\title{
Nonlinear Stochastic Analysis for Lateral Vibration of Footbridge under Pedestrian Narrowband Excitation
}

\author{
Jia Bu-yu, Yu Xiao-lin, Yan Quan-sheng, and Yang Zheng \\ School of Civil Engineering and Transportation, South China University of Technology, Guangzhou, China \\ Correspondence should be addressed to Yu Xiao-lin; xlyul@scut.edu.cn
}

Received 11 March 2017; Revised 14 May 2017; Accepted 7 June 2017; Published 9 July 2017

Academic Editor: Roman Lewandowski

Copyright (C) 2017 Jia Bu-yu et al. This is an open access article distributed under the Creative Commons Attribution License, which permits unrestricted use, distribution, and reproduction in any medium, provided the original work is properly cited.

\begin{abstract}
During the lateral vibration of footbridge, the pedestrian lateral load shows two distinct features: one is the vibration-dependency; another is the narrowband randomness caused by the variability between two subsequent walking steps. In this case, the lateral vibration of footbridge is actually a complicated, nonlinear stochastic system. In this paper, a novel nonlinear stochastic model for lateral vibration of footbridge is proposed, in which a velocity-dependent load model developed from Nakamura model is adopted to represent the pedestrian-bridge interaction and the narrowband stochastic characteristic is considered. The amplitude and phase involved Itô equations are established using the multiscale method. Based on the maximal Lyapunov exponent derived from these equations, the critical condition for triggering a large lateral vibration can be obtained by solving the stability problem. The validity of the proposed method is confirmed, based on performing the case studies of two bridges. Meanwhile, through parameter analysis, the influences of several crucial parameters on the stability of vibration are discussed.
\end{abstract}

\section{Introduction}

The infamous incidents of large lateral vibrations of the Solferino Bridge in 1999 and the London Millennium Bridge in 2000 highlight the divergence instability in pedestrianinduced vibration; that is, a slight increase in the number of people will cause the finite vibration of footbridge to diverge with large amplitude. Since these infamous incidents, scholars have begun to study the mechanism of these vibrations and gradually realized that the large lateral vibration of footbridge is a complicated process that may be governed by several coexisting mechanisms rather than a single one. To explain such vibration, several models have been proposed and classified into deterministic and stochastic models.

The representative deterministic models include the Fujino [1], Dallard [2], Nakamura [3], Ingólfsson [4, 5], Macdonald [6, 7], Roberts [8], Newland [9], Piccardo [10], Blekherman [11, 12], and Strogatz [13] models. The Fujino model can be regarded as a linear direct resonance model where the lateral vibrations are caused by direct resonance; that is, the pedestrian walking frequency is in resonance with the natural frequency of one or more lateral vibration modes. This model assumes that the vibration amplitude continues to increase along with the number of pedestrians, but such assumption contradicts the observed sudden divergent vibration on the Millennium Bridge. The Dallard, Nakamura, and Ingólfsson models are velocity-dependent nonlinear models where the pedestrian lateral load is assumed to be influenced by the footbridge velocity response and such influence can be described using a known model. The velocity-related terms in a velocity-dependent nonlinear model represent the external addition of negative damping to the bridge. The critical number of pedestrians needed to trigger the divergence can be obtained when the overall modal damping becomes negative. The Macdonald, Roberts, and Newland models couple pedestrian motion with bridge vibration as well as considering the influence of footbridge vibration on the pedestrian lateral load. However, such influence is not described by a known empirical parameter model but rather by coupled equations that include pedestrian motion and bridge vibration. The Piccardo model is a parametric resonance model that attributes the excessive pedestrian-induced lateral vibration in flexible footbridges to a parametric resonance in which the lateral natural frequency is equivalent to half of the pedestrian lateral walking frequency. The Blekherman model is an autoparametric resonance model that assumes 
that the vertical and lateral modes are coupled and that the vertical excitation energy is transferred to the lateral direction when the vertical and lateral frequencies of the footbridge show multiple relationships. The Strogatz model is a pedestrian phase synchronization model that is originally used to analyze the onset of synchronization in populations of coupled oscillators. According to this model, the initially randomly distributed walking frequencies of pedestrians will be synchronized with that of bridge if an external stimulus (e.g., vibration amplitude) is strong enough or if the step frequency is close to the vibration frequency of the bridge.

The major existent models are almost based on deterministic methods yet ignore the obvious randomness in pedestrian load. Actually, the pedestrian load is a complex stochastic process that involves large intrasubject variability from the same person and intersubject variability among different people, thereby making the pedestrian load in actual cases become significantly different from that in a deterministic case. This paper focuses on intrasubject variability. In the deterministic time domain analysis, the pedestrian load is always assumed to be a perfect periodic load that can be transformed into multiorder harmonic loads by Fourier series. However, the real consecutive pedestrian load is not a perfect periodic load but rather a narrowband stochastic process caused by intrasubject variability. If the presented load is transformed into the frequency domain, then the Fourier spectra are not distributed in discrete frequency points as a perfect periodic load but within a certain distributed width around the main harmonics, thereby leading to a reduced response. Only few stochastic models exist today, such as the Brownjohn [14], Živanović [15], Ingólfsson [4], and Racic models [16, 17]. Although the Brownjohn and Živanović models aim at the pedestrian vertical load, their modeling approaches for intrasubject randomness can also be applied to the pedestrian lateral load. These two models, which can express the pedestrian load through their Power Spectral Density (PSD), allow for the evaluation of the variance of the structural displacement and acceleration response. However, these models do not account for the influence of bridge vibration on pedestrian load. The Ingólfsson model aims at the pedestrian lateral load, in which motion-induced forces, including equivalent damping and inertia forces, are quantified through random coefficients that are generated from a discrete-time Gaussian Markov process. This model also considers the randomness of body weight, walking frequency, step length, walking speed, and arrival time among different people to represent the intersubject randomness. The critical number of pedestrians can be predicted through the criteria of the initial zero-crossing of overall damping and the first exceedance of the acceleration threshold, although this model only generates numerical results instead of an algebraic solution. The Racic model focuses on the multiple Gaussian fitting of the Autospectral Densities (ASD) of pedestrian lateral excitation, by which the variations of amplitude and phase during the real pedestrian lateral excitation process can be effectively reproduced. However, this model employs too many empirical parameters to fit numerous Gaussian functions.

The tests performed on the Millennium Bridge show that the pedestrian lateral load depends on the bridge velocity.
In the Dallard model, the pedestrian lateral load is thought to be proportional to bridge velocity, thereby indicating that the bridge vibration will reach infinity as the lateral load increases. This result contradicts the fact that pedestrians will either reduce their walking speed or completely stop when the bridge velocity becomes large. The Nakamura model starts from the Dallard model but introduces a nonlinear velocity-dependent function to represent the self-limiting nature of pedestrian action (i.e., the pedestrian response to bridge vibration will saturate under a large bridge velocity). Given that the nonlinear velocity-dependent function adopts a fraction form and includes an absolute value, this model cannot be used for conveniently implementing an algebraic analysis. Another downside to this model is that the pedestrian lateral load does not contain harmonic terms, thereby indicating that it is independent of walking frequency (i.e., the effect of the variation of pedestrian walking frequency on vibration is ignored). Inspired by the Nakamura model, this paper adopts a nonlinear velocity-dependent model to represent the pedestrian-bridge interaction but replaces the fraction function with a hyperbolic tangent function, like the way that is proposed by Bin and Weiping [18]. Meanwhile, given that the pedestrian gait varies with each step (i.e., intrasubject variability), the pedestrian lateral load is treated as a narrowband stochastic excitation process to take into account the intrasubject variability. Unlike the numerical simulation method that requires a large number of calculations, the proposed model is established in a theoretic framework of nonlinear stochastic vibration. Based on the Itô equations that are derived using the multiscale method, the critical condition for triggering a large lateral vibration is obtained according to the maximal Lyapunov exponent, whose sign is used to indicate stability or instability. The effectiveness of the proposed model is then confirmed through its applications on the Millennium Bridge (M-Bridge) and the Passerelle Simone de Beauvoir Bridge (P-Bridge), and the influences of some crucial parameters on the stability of vibration are discussed.

\section{Model for the Lateral Vibration of Footbridge}

2.1. Stochastic Model of Pedestrian Lateral Load. The Nakamura model is taken as the basic model to represent the pedestrian lateral load. However, due to the lack of harmonic term, the Nakamura model can only consider the case of direct resonance, while it fails to consider the effect of the pedestrian frequency distribution on vibration. Obviously, such model cannot give a justified explanation for the large lateral vibration of lower frequency bridge modes around $0.5 \mathrm{~Hz}$. To address this concern, a harmonic term (it becomes a stochastic excitation process when the intrasubject randomness is considered, more on this later) is introduced into the load model to refine the Nakamura model.

Then the lateral load per unit length exerted by pedestrians can be defined as follows:

$$
f_{c}(t)=m_{p} g d_{0} \rho_{0} G(y, \dot{y}, \ddot{y}) \xi(t),
$$




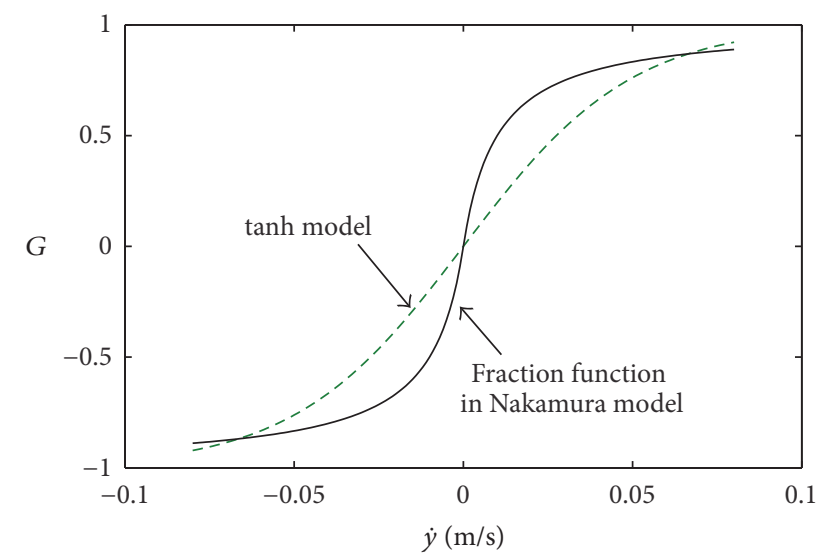

FIGURE 1: Relationship between $G$ and $\dot{y}$ under the hyperbolic tangent function and the fraction function in Nakamura model.

where $m_{p}=N m_{\mathrm{ps}} / L$ with $N$ being the number of pedestrians and $m_{\mathrm{ps}}$ being the single pedestrian mass, based on the assumption that the pedestrians are assumed to be uniformly distributed along the bridge length $L . g$ is the acceleration of gravity, and $d_{0}$ is the dynamic loading factor of the first harmonic that takes a value of 0.04 according to $[5,14,19]$ (this paper considers only the first harmonic and ignores the contribution from other high harmonics [20]). $\rho_{0}$ denotes the synchronization coefficient that takes a value of 0.2 according to $[1,3], G(y, \dot{y}, \ddot{y})$ is the vibration-dependent function that describes the interaction between pedestrian and footbridge vibration response (i.e., the displacement $y$, velocity $\dot{y}$, and acceleration $\ddot{y})$, and $\xi(t)$ denotes the stochastic excitation process (or the harmonic function if the deterministic periodic load is considered).

As mentioned previously, it is not convenient to implement an algebraic analysis for the Nakamura model due to its assumption that the velocity-dependent function $G$ has a fraction form including an absolute value. Therefore, the function of $G$ is expressed by the following velocitydependent hyperbolic tangent function proposed by [18]:

$$
G=G(\dot{y})=\tanh (c \dot{y}) .
$$

Figure 1 shows the comparison result of $G$ between the hyperbolic tangent function $(c=25 \mathrm{~s} / \mathrm{m})$ and the fraction function in the Nakamura model.

By employing the Taylor series expansion and ignoring those terms with orders of larger than three, $\tanh (c \dot{y})$ is rewritten as follows:

$$
\tanh (c \dot{y})=c \dot{y}-\frac{c^{3}}{3} \dot{y}^{3}+O\left(\dot{y}^{5}\right) .
$$

Substituting (2) and (3) into (1) yields

$$
f_{c}(t)=m_{p}(x) g d_{0} \rho_{0}\left(c \dot{y}-\frac{c^{3}}{3} \dot{y}^{3}\right) \xi(t) .
$$

Given that intrasubject variability is considered, $\xi(t)$ is characterized by a narrowband where PSD obtained from the experiment is often described by a Gaussian-shaped function.
The stochastic process with such kind of PSD can be discretized by using some spectral representation methods. The method proposed by Shinozuka [21] can be used for the numerical simulation, but this method is not suitable for algebraic theoretic analyses. Therefore, an equivalent PSD conversion method is proposed (see Appendix for details), by which the Gaussian-shaped PSD of pedestrian lateral load from existing experiment made by Pizzimenti [22] is converted into a rational form that is expressed as follows by the harmonic function with random frequency and phase:

$$
\xi(t)=\sigma_{\xi} \cos \left(\omega_{p} t+\delta B(t)+\Phi\right),
$$

where $\sigma_{\xi}$ denotes the excitation intensity, $\omega_{p}$ denotes the central pedestrian walking frequency, $\delta$ denotes the random disturbance intensity of frequency, $B(t)$ denotes the standard Weiner process, and $\Phi$ denotes the uniformly distributed phase within the interval of $[0,2 \pi)$. By employing the equivalent conversion method, the final equivalent harmonic function that represents the pedestrian lateral excitation process is obtained as follows:

$$
\begin{aligned}
\xi(t) & =\sigma_{\xi} \bar{\xi}(t)=\sigma_{F} h \bar{\xi}(t) \\
& =\sigma_{F} h \cos \left(\omega_{p} t+\delta B(t)+\Phi\right), \\
h & =\sqrt{\frac{2 \pi \sqrt{2 \pi} a_{s}}{\sqrt{2-b_{s}^{2}}}}, \delta=\sqrt{\frac{2 \omega_{p} b_{s}}{\sqrt{2-b_{s}^{2}}}}
\end{aligned}
$$

where $\sigma_{F}$ corresponds to the quantified coefficient of $\xi(t)$ in (4), $h$ is regarded as the externally added excitation intensity factor during the conversion, and $\bar{\xi}(t)$ denotes the pedestrian lateral stochastic process with unit excitation intensity. $a_{s}=$ 0.9 and $b_{s}=0.043$ are the fitting parameters obtained from the test results of Pizzimenti and Ricciardelli [22].

2.2. Equations of Footbridge Lateral Vibration. By considering the footbridge as an Euler Bernoulli beam, its lateral motion can be expressed as follows:

$$
\begin{aligned}
m_{s}(x) & \frac{\partial^{2} y(x, t)}{\partial t^{2}}+c_{s}(x) \frac{\partial y(x, t)}{\partial t} \\
+ & \frac{\partial^{2}}{\partial x^{2}}\left[E I(x) \frac{\partial^{2} y(x, t)}{\partial x^{2}}\right]=f_{c}(t),
\end{aligned}
$$

where $m_{s}(x), c_{s}(x)$, and $E I(x)$ are bridge mass per unit length, bridge damping per unit length, and bridge bending stiffness, respectively. By assuming that the bridge damping is proportional, (7) can be decoupled into modal differential equations. The first-order mode $y(x, t)=\varphi(x) q(t)$ is only considered, where the mode shape function is considered as $\phi(x)=\sin (\pi x / L)$. Notably, the central span of the MBridge (one of the study objects as discussed later) also has a higher lateral mode, second lateral mode with a frequency being around $1 \mathrm{~Hz}$, which could activate a direct resonance. However, its shape is skew-symmetric and cannot produce large vibration at midspan unless the pedestrian mass is not 
uniformly distributed. The corresponding modal differential equation is expressed as follows:

$$
\begin{aligned}
\ddot{q}(t) & +2 \omega_{s} \zeta \dot{q}(t)+\omega_{s}^{2} q(t)=\frac{1}{M_{s}} \int_{0}^{L} f_{c}(t) \varphi(x) \mathrm{d} x \\
= & F(t),
\end{aligned}
$$

where $q(t), \omega_{s}, \zeta, \varphi(x), M_{s}$, and $F(t)$ are the modal displacement, angular frequency, modal damping ratio, mode shape, modal mass, and mass normalized modal load, respectively. According to (4) and (6), the mass normalized modal load $F(t)$ is expressed as follows:

$$
\begin{aligned}
F(t)= & \frac{d_{0} \rho_{0} g c h}{M_{s}}\left[\int_{0}^{L} m_{p}(x) \varphi(x)^{2} \mathrm{~d} x\right] \dot{q} \bar{\xi}(t) \\
& -\frac{d_{0} \rho_{0} g c^{3} h}{3 M_{s}}\left[\int_{0}^{L} m_{p}(x) \varphi(x)^{4} \mathrm{~d} x\right] \dot{q}^{3} \bar{\xi}(t) .
\end{aligned}
$$

By setting $g_{1}=\left(d_{0} \rho_{0} g c h / M_{s}\right)\left[\int_{0}^{L} m_{p}(x) \varphi(x)^{2} \mathrm{~d} x\right]$ and $g_{2}=\left(d_{0} \rho_{0} g c^{3} h / 3 M_{s}\right)\left[\int_{0}^{L} m_{p}(x) \varphi(x)^{4} \mathrm{~d} x\right]$, (9) becomes

$$
F(t)=g_{1} \dot{q} \bar{\xi}(t)-g_{2} \dot{q}^{3} \bar{\xi}(t) .
$$

By substituting (10) into (8), the motion equation of footbridge is expressed as follows:

$$
\ddot{q}+2 \omega_{s} \zeta \dot{q}+\omega_{s}^{2} q=g_{1} \dot{q} \bar{\xi}(t)-g_{2} \dot{q}^{3} \bar{\xi}(t) .
$$

\section{Theoretical Derivation Using the Multiscale Method}

3.1. Multiscale Method. Multiplying the damping term and the external excitation term in (11) by a small parameter $\varepsilon$ yields

$$
\ddot{q}+2 \varepsilon \omega_{s} \zeta \dot{q}+\omega_{s}^{2} q=\varepsilon g_{1} \dot{q} \bar{\xi}(t)-\varepsilon g_{2} \dot{q}^{3} \bar{\xi}(t) .
$$

The multiscale method is used to solve (12). The firstorder approximate with two time scales is expressed as follows:

$$
q\left(T_{0}, T_{1}\right)=q_{0}\left(T_{0}, T_{1}\right)+\varepsilon q_{1}\left(T_{0}, T_{1}\right)+\cdots,
$$

where $T_{0}=t$ and $T_{1}=\varepsilon t$. Considering the differential operators of $D_{0}=\partial / \partial T_{0}$ and $D_{1}=\partial / \partial T_{1}$, the following equation is obtained:

$$
\begin{aligned}
\frac{d}{d t} & =D_{0}+\varepsilon D_{0}+\cdots, \\
\frac{d^{2}}{d t^{2}} & =D_{0}^{2}+2 \varepsilon D_{0} D_{1}+\cdots .
\end{aligned}
$$

Substituting (13) and (14) into (12) yields

$$
\begin{aligned}
D_{0} q_{0}+\omega_{s}^{2} q_{0}= & 0 \\
D_{0}^{2} q_{1}+\omega_{s}^{2} q_{1}= & -2 D_{0} D_{1} q_{0}-2 \omega_{s} \zeta D_{0} q_{0}+g_{1} D_{0} q_{0} \bar{\xi}(t) \\
& -g_{2}\left(D_{0} q_{0}\right)^{3} \bar{\xi}(t) .
\end{aligned}
$$

The solution to (15) can be written as follows:

$$
\begin{aligned}
q_{0}\left(T_{0}, T_{1}\right)= & A\left(T_{1}\right) \exp \left(i w_{s} T_{0}\right) \\
& +\bar{A}\left(T_{1}\right) \exp \left(-i w_{s} T_{0}\right),
\end{aligned}
$$

where $A\left(T_{1}\right)$ denotes the complex functions with respect to $T_{1}$ and $\bar{A}\left(T_{1}\right)$ denotes the complex conjugate of $A\left(T_{1}\right)$.

Substituting (6) and (17) into (16) yields

$$
\begin{aligned}
& D_{0}^{2} q_{1}+\omega_{s}^{2} q_{1}=-i 2 \omega_{s} A^{\prime} \exp \left(i \omega_{s} T_{0}\right)-i 2 \omega_{s}^{2} \zeta A \\
& \cdot \exp \left(i \omega_{s} T_{0}\right)+0.5 g_{1}\left\{i \omega_{s} A\right. \\
& \cdot \exp \left[i\left(\omega_{p}+\omega_{s}\right) T_{0}+i \widehat{\delta} B\left(T_{1}\right)+i \Phi\right]-i \omega_{s} \bar{A} \\
& \left.\cdot \exp \left[i\left(\omega_{p}-\omega_{s}\right) T_{0}+i \widehat{\delta} B\left(T_{1}\right)+i \Phi\right]\right\} \\
& -0.5 g_{2}\left\{-i \omega_{s}^{3} A^{3}\right. \\
& \cdot \exp \left[i\left(\omega_{p}+3 \omega_{s}\right) T_{0}+i \widehat{\delta} B\left(T_{1}\right)+i \Phi\right]-i \omega_{s}^{3} \bar{A}^{3} \\
& \cdot \exp \left[-i\left(\omega_{p}-3 \omega_{s}\right) T_{0}-i \widehat{\delta} B\left(T_{1}\right)-i \Phi\right] \\
& +i 3 \omega_{s}^{3} A^{2} \bar{A} \exp \left[i\left(\omega_{p}+\omega_{s}\right) T_{0}+i \widehat{\delta} B\left(T_{1}\right)+i \Phi\right] \\
& \left.-i 3 \omega_{s}^{3} A \bar{A}^{2} \exp \left[i\left(\omega_{p}-\omega_{s}\right) T_{0}+i \widehat{\delta} B\left(T_{1}\right)+i \Phi\right]\right\} \\
& +\operatorname{cc},
\end{aligned}
$$

where $A^{\prime}$ denotes the derivative with respect to $T_{1}$, cc denotes the complex conjugate of all preceding terms on the right side of (18), and $\widehat{\delta}=\delta / \sqrt{\varepsilon}$ because $\delta B(t)=\delta B(\varepsilon t) / \sqrt{\varepsilon}=\widehat{\delta} B\left(T_{1}\right)$.

On the basis of (18), a parametric resonance will occur when $\omega_{p} \approx 2 \omega_{s}$, whereas a forced vibration will occur when $\omega_{p} \approx \omega_{s}$. Dallard et al.s [23] tests on the London Millennium Bridge revealed that the first lateral mode of the central span with a frequency of $0.48 \mathrm{~Hz}$ was excited, which indicates an approximate $1: 2$ relationship between the mode and walking frequency. In the normal case, except for the heavy congestion, crowd density has a limited influence on the pedestrian walking frequency, and pedestrians are unlikely to slow down their walking frequency to an unusual level. In this paper, a normal walking with a frequency of approximately $1.0 \mathrm{~Hz}$ (i.e., approximately twice the walking frequency) is assumed, and the effect of synchronization is also considered. Given the above consideration, it seems that the parametric resonance rather than other mechanisms (e.g., the forced vibration [1] and the nonsynchronization model $[4,6])$ is reasonable for explaining the large vibration of the first lateral mode of the central span of the Millennium Bridge. Thus, only $\omega_{p} \approx 2 \omega_{s}$ is considered in this paper. Introducing a detuning parameter $\varepsilon \sigma$ yields the following:

$$
\omega_{p}=2 \omega_{s}+\varepsilon \sigma
$$


Eliminating the secular terms in (18) yields

$$
\begin{aligned}
& -i 2 \omega_{s} A^{\prime}-i 2 \omega_{s}^{2} \zeta A \\
& -i \frac{g_{1}}{2} \omega_{s} \bar{A} \exp \left[i \sigma T_{1}+i \widehat{\delta} B\left(T_{1}\right)+i \Phi\right] \\
& +i \frac{g_{2}}{2} \omega_{s}^{2} A^{3} \exp \left[-i \sigma T_{1}-i \widehat{\delta} B\left(T_{1}\right)-i \Phi\right] \\
& +i \frac{3 g_{2}}{2} \omega_{s}^{3} A \bar{A}^{2} \exp \left[i \sigma T_{1}+i \widehat{\delta} B\left(T_{1}\right)+i \Phi\right]=0 .
\end{aligned}
$$

By setting $A=(1 / 2) a\left(T_{1}\right) \exp \left[i \varphi\left(T_{1}\right)\right]$ and $\Delta\left(T_{1}\right)=\sigma T_{1}+$ $\widehat{\delta} B\left(T_{1}\right)+\Phi-2 \varphi\left(T_{1}\right),(20)$ becomes

$$
\begin{aligned}
& -i \omega_{s} a^{\prime}+\omega_{s} a \varphi^{\prime}-i \omega_{s}^{2} a \zeta \\
& +\left(i \frac{3 g_{2}}{16} \omega_{s}^{3} a^{3}-i \frac{g_{1}}{4} \omega_{s} a\right)[\cos \Delta+i \sin \Delta] \\
& +i \frac{g_{2}}{16} \omega_{s}^{3} a^{3}[\cos \Delta-i \sin \Delta]=0 .
\end{aligned}
$$

Separating (21) into real and imaginary parts yields

$$
\begin{aligned}
a^{\prime} & =-\omega_{s} \zeta a-\frac{g_{1}}{4} a \cos \Delta+\frac{g_{2}}{4} \omega_{s}^{2} a^{3} \cos \Delta, \\
a \varphi^{\prime} & =-\frac{g_{1}}{4} a \sin \Delta+\frac{g_{2}}{8} \omega_{s}^{2} a^{3} \sin \Delta .
\end{aligned}
$$
follows:

As $2 a \varphi^{\prime}=a \sigma+a \widehat{\delta} B^{\prime}\left(T_{1}\right)-a \Delta^{\prime},(22)$ can be rewritten as

$$
\begin{aligned}
a^{\prime}= & -\omega_{s} \zeta a-\frac{g_{1}}{4} a \cos \Delta+\frac{g_{2}}{4} \omega_{s}^{2} a^{3} \cos \Delta, \\
\Delta^{\prime}= & \left(\omega_{p}-2 \omega_{s}\right)+\frac{g_{1}}{2} \sin \Delta-\frac{g_{2}}{4} \omega_{s}^{2} a^{2} \sin \Delta \\
& +\widehat{\delta} B^{\prime}(t) .
\end{aligned}
$$

3.2. Stability Analysis. Implementing a linear operation with regard to the derived coefficients in (23) at $a=0$ yields

$$
\begin{aligned}
\mathrm{d} a & =\widehat{m}_{1} \mathrm{~d} t=\left[-\omega_{s} \zeta-\frac{g_{1} \cos \Delta}{4}\right] a \mathrm{~d} t, \\
\mathrm{~d} \Delta & =\widehat{m}_{2} \mathrm{~d} t+\delta \mathrm{d} B(t) \\
& =\left(\omega_{p}-2 \omega_{s}+\frac{g_{1} \sin \Delta}{2}\right) \mathrm{d} t+\delta \mathrm{d} B(t) .
\end{aligned}
$$

By introducing a new variable $\Upsilon=\ln a$, (24) can be rewritten as follows:

$$
\begin{aligned}
& \mathrm{d} \Upsilon=\left(-\omega_{s} \zeta-\frac{g_{1} \cos \Delta}{4}\right) \mathrm{d} t \\
& \mathrm{~d} \Delta=\left(\omega_{p}-2 \omega_{s}+\frac{g_{1} \sin \Delta}{2}\right) \mathrm{d} t+\delta \mathrm{d} B(t) .
\end{aligned}
$$

Given that $\Delta(t)$ is the ergodic Markov process in the interval of $[0,2 \pi]$, its invariant measure represented by the stationary probability density function $p(\Delta)$ satisfies the following stationary Fokker-Planck-Kolmogorov equation:

$$
\frac{d^{2} p}{d \Delta^{2}}-\frac{d}{d \Delta}[(\bar{\sigma}+\bar{g} \sin \Delta) p]=0,
$$

where $\bar{\sigma}=2\left(\omega_{p}-2 \omega_{s}\right) / \delta^{2}$ and $\bar{g}=g_{1} / \delta^{2} \cdot p(\Delta)$ satisfies the periodicity condition $p(\Delta)=p(\Delta+2 \pi)$ and the probability normalization condition $\int_{0}^{2 \pi} p(\Delta) \mathrm{d} \Delta=1$, which can be solved as follows:

$$
\begin{aligned}
p(\Delta)= & C \exp (\bar{\sigma} \Delta-\bar{g} \cos \Delta) \\
& \cdot \int_{\Delta}^{\Delta+2 \pi} \exp (-\bar{\sigma} \tau+\bar{g} \cos \Delta) d \tau,
\end{aligned}
$$

where $C$ is the probability normalization constant. According to Oseledec's multiplicative ergodic theorem, for any initial value $\left(a_{0}, \Delta_{0}\right)$, the Lyapunov exponent $\lambda\left(a_{0}, \Delta_{0}\right)$ of the solution $a\left(t ; a_{0}, \Delta_{0}\right)$ of $(24)$ can be defined as follows:

$$
\lambda\left(a_{0}, \Delta_{0}\right)=\lim _{t \rightarrow \infty} \frac{1}{t} \ln \left|a\left(t ; a_{0}, \Delta_{0}\right)\right|, \quad \text { w.p. } 1,
$$

where w.p. 1 means with probability one. Given that $\lambda\left(a_{0}, \Delta_{0}\right)$ only has two different values, the stability of the trivial solution of (24) depends on the maximal Lyapunov exponent $\lambda_{\text {max }}$. The trivial solution of (24) is stable with probability one when $\lambda_{\max }<0$ and unstable with probability one when $\lambda_{\max }>0$, thereby suggesting that the boundary condition between the stability and instability of (12) can be approximately represented by $\lambda_{\max }=0$. According to [24], the maximal Lyapunov exponent $\lambda_{\max }$ can be obtained as follows by considering the ergodicity of $\Delta(t)$ :

$$
\begin{aligned}
\lambda_{\max } & =\lim _{t \rightarrow \infty} \frac{1}{t}\left|\frac{a(t)}{a(0)}\right|=\lim _{t \rightarrow \infty} \frac{1}{t}[\Upsilon(t)-\Upsilon(0)] \\
& =-\omega_{s} \zeta-\lim _{t \rightarrow \infty} \frac{1}{t} \int_{0}^{t} \frac{g_{1}}{4} \cos \Delta(\tau) d \tau \\
& =-\omega_{s} \zeta-\frac{g_{1}}{4} E[\cos \Delta] \\
& =-\omega_{s} \zeta-\frac{g_{1}}{4} \int_{0}^{2 \pi} p(\Delta) \cos \Delta d \Delta,
\end{aligned}
$$

where $E[*]$ denotes the expected value operator.

\section{Case Study}

4.1. Critical Number of Pedestrians. The central span of the M-Bridge (a shallow suspension bridge located in London) and the central span of the P-Bridge (a combined shallow arch bridge located in Paris) are selected to be the objects of the present study. According to previous works [2, 25, 26], the structural parameters of these bridges as well as the other common involved parameters are listed in Table 1.

According to $\lambda_{\max }=0$, the critical number of pedestrians $N_{\lim }\left(N=m_{p} L / m_{\mathrm{ps}}\right)$ needed to trigger the unstable lateral vibration of footbridge can be obtained. Figure 2 presents 
TABLE 1: Values of parameters.

\begin{tabular}{lccccccccc}
\hline Bridge & $\begin{array}{c}\text { Length } \\
(\mathrm{m})\end{array}$ & $\begin{array}{c}\text { First } \\
\text { frequency } \\
(\mathrm{Hz})\end{array}$ & $\begin{array}{c}\text { Bridge } \\
\text { mass } / L \\
(\mathrm{~kg} / \mathrm{m})\end{array}$ & $\begin{array}{c}\text { Damping } \\
\text { ratio }(\%)\end{array}$ & $\begin{array}{c}\text { Dynamic } \\
\text { loading } \\
\text { factor }\end{array}$ & $\begin{array}{c}\text { Synchronization } \\
\text { coefficient }\end{array}$ & $\begin{array}{c}\text { Velocity } \\
\text { proportional } \\
\text { coefficient } \\
(\mathrm{s} / \mathrm{m})\end{array}$ & $\begin{array}{c}\text { Fitting } \\
\text { parameters in } \\
\text { PSD }\end{array}$ & $\begin{array}{c}\text { Single } \\
\text { pedestrian } \\
\text { mass }(\mathrm{kg})\end{array}$ \\
\hline M-Bridge & 144 & 0.48 & 2000 & 0.7 & 0.04 & 0.2 & $\rho_{0}$ & 25 & $0.9,0.043$ \\
P-Bridge & 190 & 0.56 & 3420 & 0.77 & $f_{s}$ & $\zeta$ & $d_{0}$ & & 70 \\
\hline
\end{tabular}

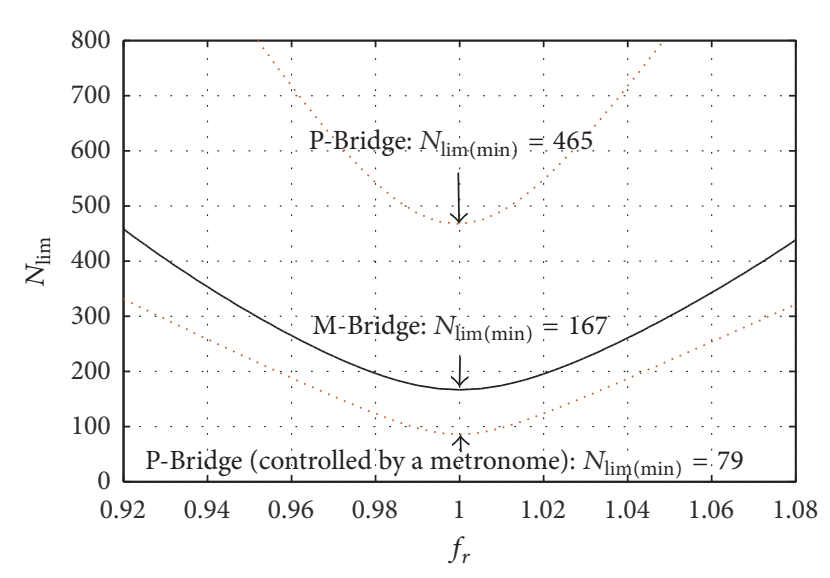

FIGURE 2: Critical number of pedestrians $N_{\text {lim }}$ as a function of the frequency ratio $f_{r}$ for the two bridges.

the critical number of pedestrians as a function of the frequency ratio between walking frequency and doubled bridge frequency $\left(f_{r}=f_{p} / 2 f_{s}\right)$ for these two bridges. Under the worst condition $\left(f_{r}=1\right)$, the minimal value of $N_{\text {lim }}$ for the central span of the M-Bridge is 167, which is consistent with the observations made on the M-Bridge [23]. Based on the statements from [25], more than 400 people are needed to trigger the instable vibration of the PBridge; however, the controlled walking tests revealed that an instable vibration with a large amplitude up to $60 \mathrm{~mm}$ was reached when a group of 60 people walked in step using a metronome to control the walking frequency. It is conceivable that the crowd controlled by a metronome will have a high synchronization, and each pedestrian will have a low intrasubject variability. Therefore, the synchronization coefficient is assumed as 0.8 , and the value of $b_{s}$ used to represent the intrasubject variability (the role of $b_{s}$ will be discussed later) is assumed as half of the normal case. Notably, the assumption lacks the support of available data, but it appears at least justifiable in terms of qualitative aspect. The dotted curves in Figure 2 show that the results from the proposed model match well with those actual observations on the P-Bridge. The well matched comparative results confirm the effectiveness of the proposed method.

4.2. Parameter Analysis. The frequency distribution, number of pedestrians, and random disturbance intensity are taken as the crucial parameters, and their influences on the stability of the central span of the M-Bridge are discussed.
Figure 3 presents the maximal Lyapunov exponent $\lambda_{\max }$ as a function of the number of pedestrians $N$ and the frequency ratio $f_{r}=f_{p} / 2 f_{s}$. Based on the mesh surface (Figure 3(a)), $\lambda_{\max }$ increases along with the number of pedestrians and reaches its peak when the walking frequency becomes equivalent to the doubled first lateral frequency $\left(f_{r}=1\right)$. In such case, the lateral vibration of bridge will be in its worst state (lowest stability). To better illustrate the influences of $N$ and $f_{r}$ on $\lambda_{\text {max }}$, Figure $3(\mathrm{~b})$ presents the curve of $\lambda_{\text {max }}-f_{r}$ under different fixed $N$, while Figure $3(\mathrm{c})$ presents the curve of $\lambda_{\max }-N$ under different fixed $f_{r}$. Figure 3(b) shows that when $N$ is relatively small, such as $N=100$ or $N=150, \lambda_{\max }$ will never exceed zero at any value of $f_{r}$. By contrast, when $N$ is large, such as $N=200$ or $N=250, \lambda_{\max }$ exceeds zero in the area where $f_{p}$ is close to $2 f_{s}$. These results reveal that the stability depends on the number of pedestrians. With a large number of pedestrians, the stability becomes sensitive to frequency distribution. However, with a small number of pedestrians, the lateral vibration of the bridge will always remain stable even if the frequency distribution is within the region of parametric resonance. Figure 3(c) shows that the sensitivity of the stability to the number of pedestrians also depends on the frequency distribution. When parametric resonance happens $\left(f_{r}=1\right)$, the stability decreases rapidly along with the number of pedestrians. When the central lateral walking frequency is far from the doubled first lateral frequency of the bridge (e.g., $f_{r}=1.1$ ), the stability is only slightly influenced by the number of pedestrians, and the state remains stable even when the number of pedestrians is considerably large.

Through the contour of $\lambda_{\max }$ as shown in Figure 4, the regions of stability and instability are obtained by taking $N$ and $f_{r}$ as the parameters. The stability region appears as a concave shape, suggesting that when the number of pedestrians is less than the value at the lowest point (i.e., 167), the lateral vibration of the bridge will always be stable regardless of how the frequency is distributed, which is consistent with the observations from Figure 3.

The influence of the randomness in the pedestrian lateral excitation on the stability is also considered in the parametric analysis of the random disturbance intensity of frequency $\delta$. From (6), it can be known that $\delta$ can be further represented by the parameter $b_{s}$ ( $\delta$ monotonically increases with $b_{s}$ ).

Figure 5 presents $\lambda_{\max }$ as a function of $b_{s}$ and $f_{r}$ when $N=167$. Figure 5(a) shows that the peak of $\lambda_{\max }$ is located at the point of minimal $b_{s}$ and $f_{r}=1$. Figure $5(\mathrm{~b})$ shows that the random disturbance intensity also has a great influence on stability. The sensitivity of the stability to the frequency 


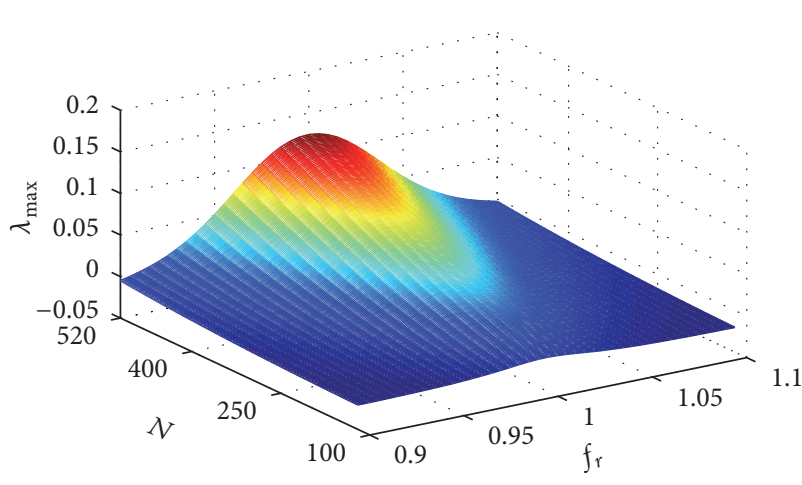

(a)

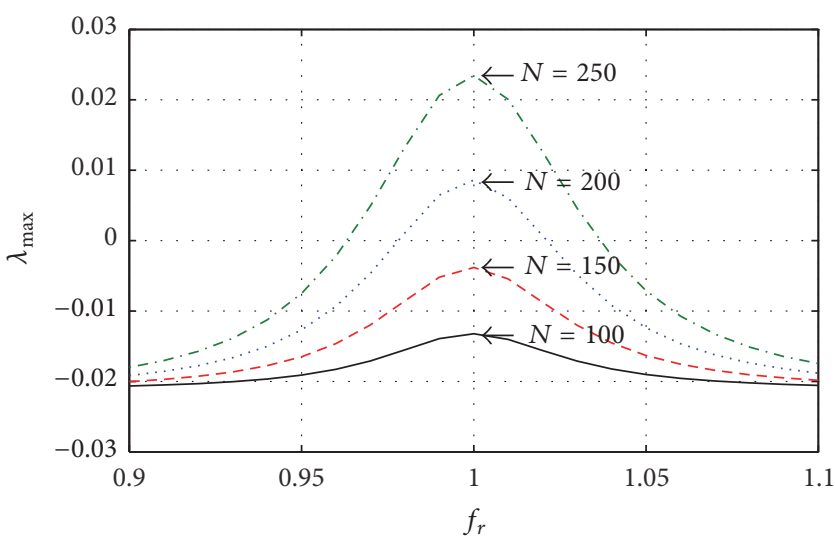

(b)

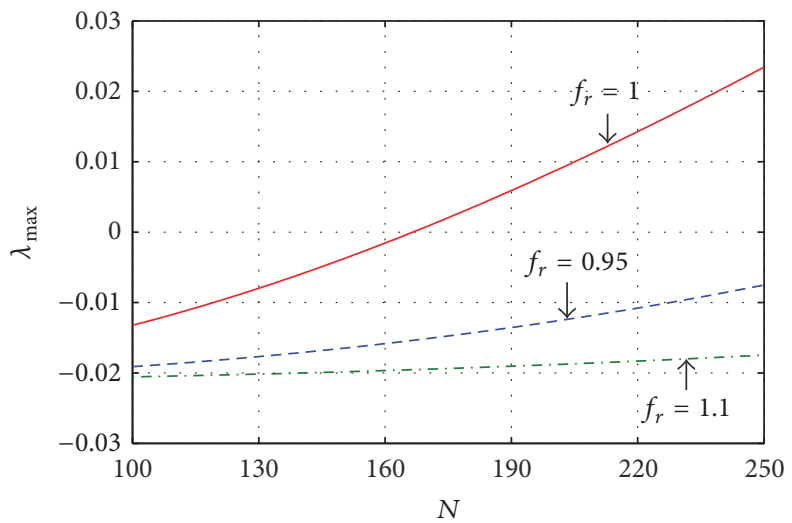

(c)

Figure 3: Maximal Lyapunov exponent $\lambda_{\max }$ as a function of the number of pedestrians $N$ and the frequency ratio $f_{r}$ : (a) mesh surface of $\lambda_{\max }-\left(N, f_{r}\right)$, (b) $\lambda_{\max }-f_{r}$ under fixed $N$, and (c) $\lambda_{\max }-N$ under fixed $f_{r}$.

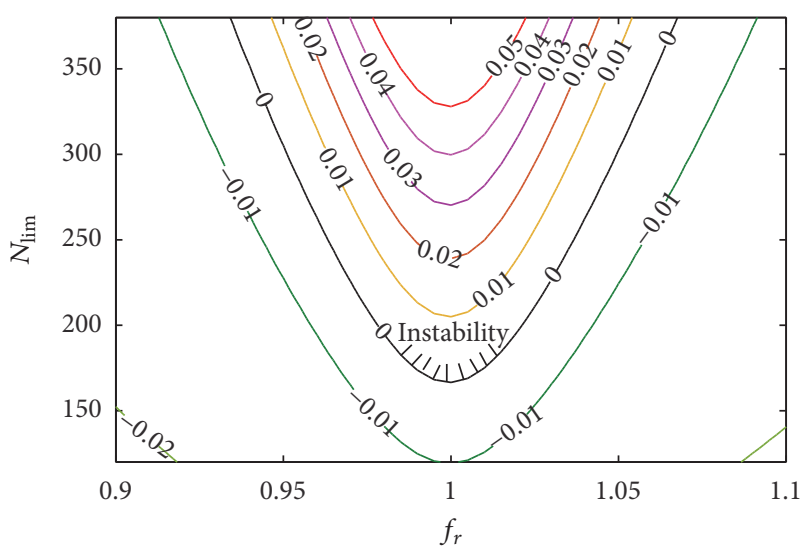

FIgURE 4: Contour of $\lambda_{\max }$ for different values of $N$ and $f_{r}$.

distribution decreases along with increasing random disturbance intensity, and the vibration always remains stable when the random disturbance intensity is relatively large (e.g., $b_{s}>0.083$ ) even if parametric resonance happens. Similar to Figure 3(c), Figure 5(c) shows that the relationship between the stability and the random disturbance intensity is determined by the frequency distribution. The stability increases rapidly along with random disturbance intensity when the central lateral walking frequency is close to the doubled first lateral frequency of the bridge. Otherwise, the stability is rarely influenced by the random disturbance intensity. Notably, when the frequency is roughly less than 0.95 or larger than 1.05 , the stability decreases slightly along with increasing random disturbance intensity.

Figure 6 presents the contour of $\lambda_{\max }$ with respect to parameters $b_{s}$ and $f_{r}$ under the case of $N=167$. Figure 6 shows a small region of instability. The lateral vibration of the bridge enters an unstable state only when $0.982<f_{r}<1.017$ and $b_{s}<0.043$.

Figure 7 presents the contours of $\lambda_{\max }$ with respect to the parameters of $N$ and $b_{s}$ under the case of $f_{r}=$ 0.9 (Figure $7(\mathrm{a})$ ) and $f_{r}=1$ (Figure $7(\mathrm{~b})$ ), respectively. The relationship between $N$ and $b_{s}$ in the contour line is approximately linear, thereby indicating that the number of pedestrians has a slight influence on the sensitivity of the stability to the random disturbance intensity, and vice versa. Meanwhile, the stability (or instability) region differs between the cases of $f_{r}=0.9$ and $f_{r}=1$. The stability tends to decrease along with increasing random disturbance intensity and the number of pedestrians when $f_{r}=0.9$ but tends to increase along with increasing random disturbance intensity and decreasing number of pedestrians when $f_{r}=1$. These 


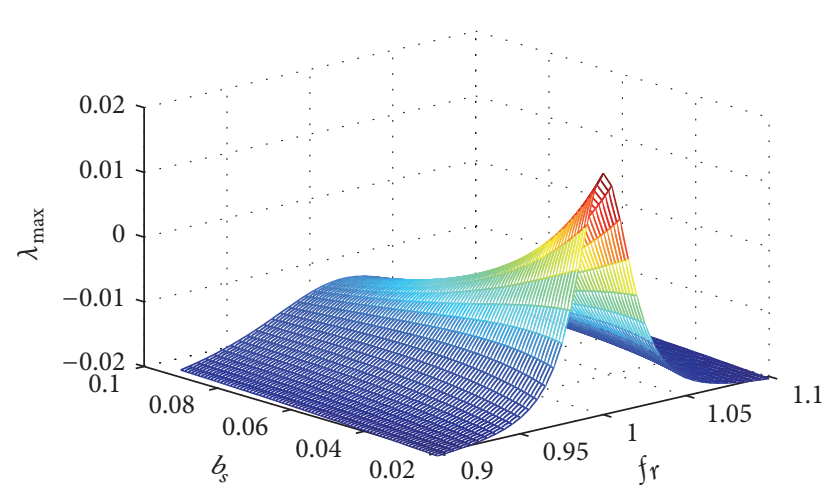

(a)

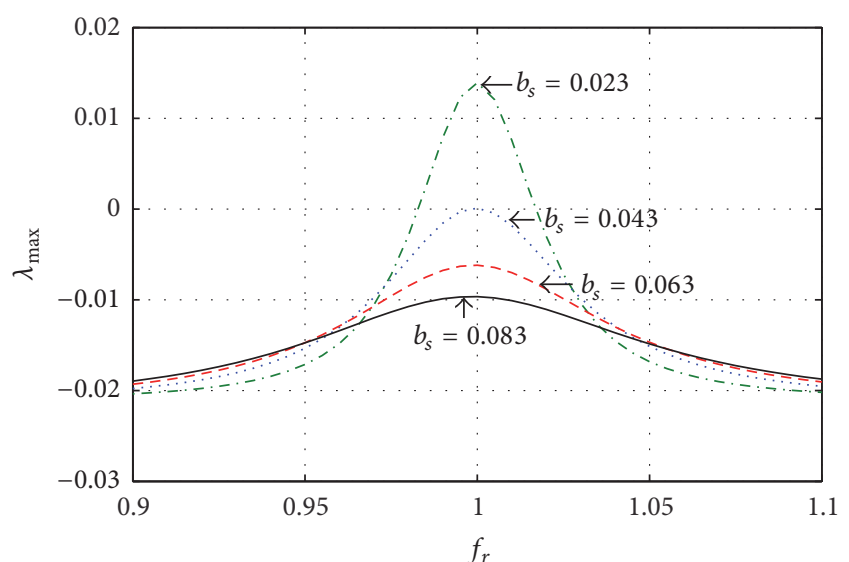

(b)

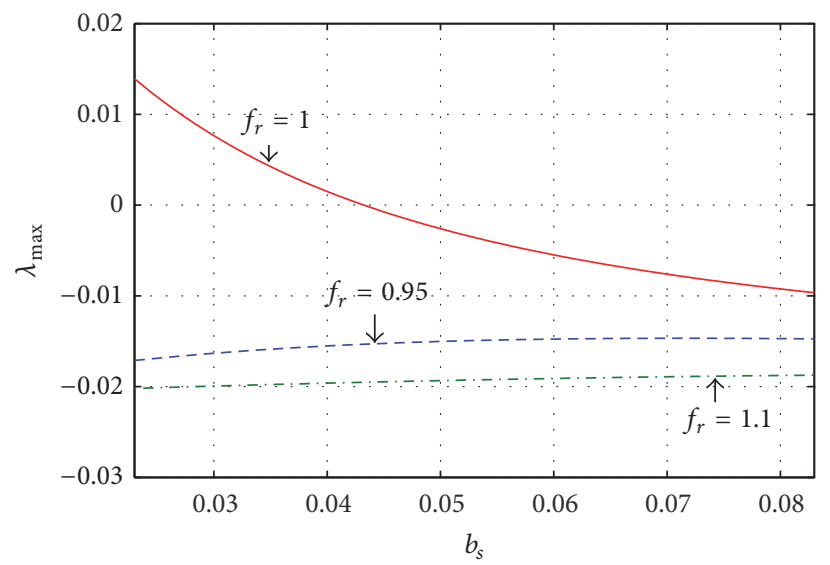

(c)

FiguRe 5: Maximal Lyapunov exponent $\lambda_{\max }$ as a function of the number of pedestrians $b_{s}$ and the frequency ratio $f_{r}$ : (a) mesh surface of $\lambda_{\max }-\left(b_{s}, f_{r}\right)$, (b) $\lambda_{\max }-f_{r}$ under fixed $b_{s}$, and (c) $\lambda_{\max }-b_{s}$ under fixed $f_{r}$.

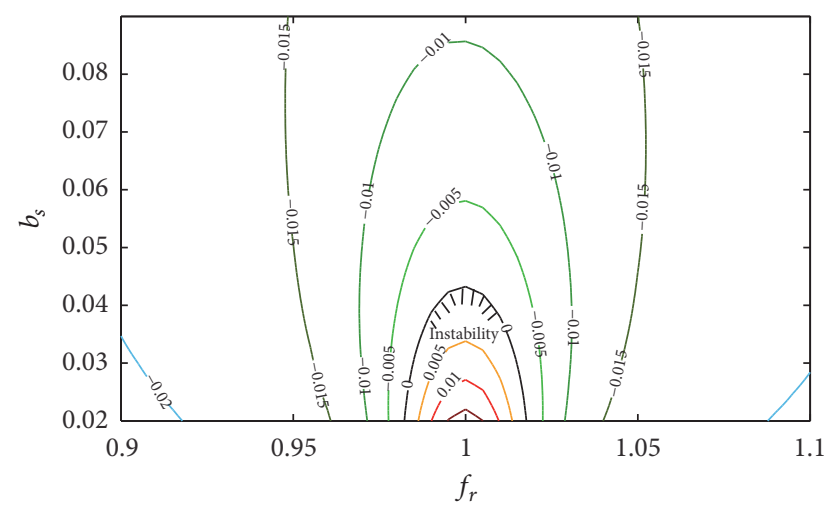

FIgURE 6: Contour of $\lambda_{\max }$ for different values of $b_{s}$ and $f_{r}$.

findings are consistent with the observations from Figures 3 and 5 .

\section{Discussions}

This paper proposes a novel theoretic nonlinear stochastic model for the lateral vibration of footbridges that adopts a velocity-dependent hyperbolic tangent function to represent the pedestrian-bridge interaction and considers the narrowband stochastic characteristic caused by the intrasubject variability. By using the multiscale method, the amplitude and phase involved Itô equations based on the stochastic parametric resonance mechanism are established, by which the critical condition for triggering the large lateral vibration of footbridges can be obtained by solving the stability problem via the identification of the sign of the maximal Lyapunov exponent.

To the authors' knowledge, the latest published models that can give a justified explanation for the large lateral vibration of lower frequency bridge modes around $0.5 \mathrm{~Hz}$ include the Piccardo, Ingólfsson, and Macdonald models. To better understand the advantages and limitations of the proposed model, all of the above models have been compared as follows.

Although the proposed model and the Piccardo model both adopt the parametric resonance mechanism to explain the large lateral vibration in a flexible bridge, these models show some distinctive differences. For instance, the former model is stochastic, while the latter model is deterministic. Moreover, the lateral pedestrian-induced force is related to 


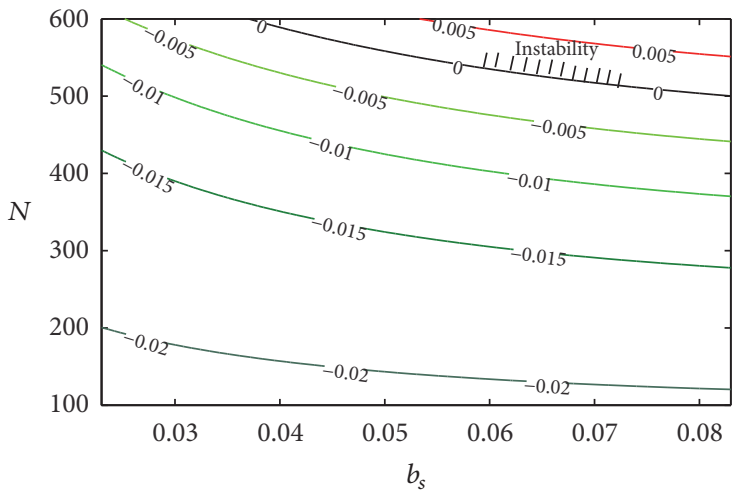

(a)

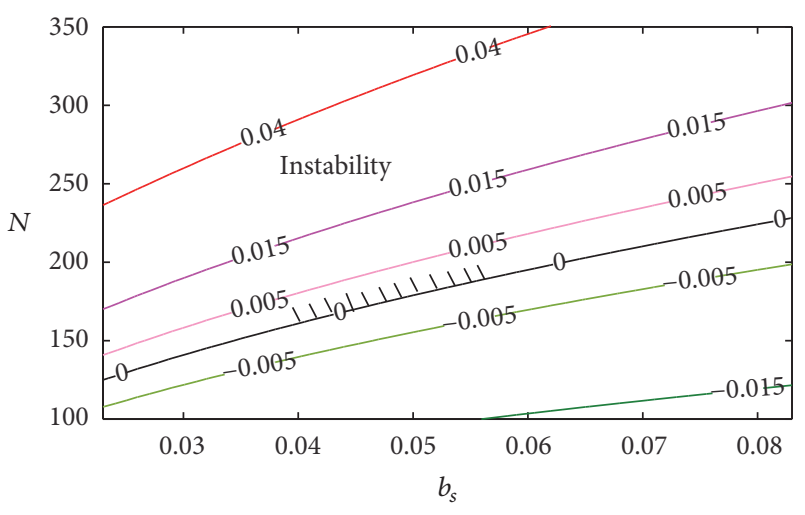

(b)

Figure 7: Contour of $\lambda_{\max }$ for different values of $N$ and $b_{s}$ : (a) $f_{r}=0.9$ and (b) $f_{r}=1$.

velocity in the former but is proportional to displacement in the latter. The comparative results about the stability criterion of these two models show that the proposed model gives a more reasonable result than the Piccardo model. The stability criterion in the Piccardo model is very sensitive to the relationship between step frequency and modal frequency. A very rapid increase in the critical number of pedestrians is also observed when $f_{p}$ deviates from $2 f_{s}$. By contrast, the results from the proposed model as shown in Figure 4 seem more reasonable than those from the Piccardo model because the sensitivity of the critical number of pedestrians to the distribution of pedestrian walking frequency has significantly decreased in the proposed model compared with that in the Piccardo model.

Unlike the pedestrian load model presented in this paper (or the Piccardo model), which is based on full-scale measurements, the Ingólfsson and Macdonald models are based on laboratory tests that are related to the latest walking tests on a moving treadmill. The Ingólfsson model is obtained from an extensive experimental campaign on a slightly modified moving treadmill of Pizzimenti and Ricciardelli [22]. The Macdonald model is an inverted pendulum model (IPM) rooted in the field of biomechanics, in which a stepping control law is adopted based on the instrumented treadmill test of Hof et al. [27]. Recently, under the IPM framework proposed by Macdonald, Carroll et al. [28, 29] rebuilt the experimental setup developed by Pizzimenti and Ricciardelli [22] and then utilized 3D motion capture equipment to analyze the features of the self-excited force caused by humanstructure interaction. Similarly, Bocian et al. [30] conducted a treadmill test based on the IPM framework by using an interactive virtual reality technology to avoid the implications of artificiality and allow for unconstrained gait in the laboratory environment. The main conclusions obtained from the laboratory tests are as follows: the velocity proportional load (or the equivalent negative damping) could be generated even though pedestrian lateral walking frequency differs from that of the bridge (i.e., synchronization is not necessary for a large vibration of footbridge); and the large vibration will be triggered when the equivalent negative damping is equal to the inherent bridge damping. In laboratory-based models (i.e., the Ingólfsson and Macdonald models), the means of adopting the velocity proportional load to reflect the effect of structure vibration on pedestrian load and of defining the stability criterion based on whether total damping is less than zero are similar to those used in this study. Additionally, a certain degree of intrasubject randomness has been observed in most of the laboratory tests, which further proves the necessity of stochastic analysis. The main difference between the pedestrian load model presented in this paper and laboratory-based models is that the former is a macroscopic model, the parameters of which are estimated via back-analysis, whereas the latter is a microscopic model, the parameters of which are obtained directly from laboratory tests. Laboratory-based models focus on a single pedestrian behavior when perturbed by structure motion through several comprehensive tests, which may provide a more precise description of the interaction between a single pedestrian and a bridge and can be generalised to other structures. However, existing laboratory-based models also exhibit insufficiencies that are worthy of discussion. These models are derived from a test in which only a single pedestrian is walking on a treadmill. That is, whether these models can be applied to crowds walking on a bridge is doubtful because the psychological differences between these two environments may affect a pedestrian's choice of gait and footfall. In fact, the behavior of a pedestrian in a crowd considerably differs from that when he/she is alone due to the effect of the surrounding crowd. For example, in a high density case, pedestrians will tend to synchronize to each other, due to the attempt to avoid foot-to-foot and shoulder-to-shoulder contact. Moreover, the aforementioned laboratory-based models typically involve an elaborate modeling process with a number of variables and equations, which require using a numerical simulation method, thereby making these models inconvenient to operate. Admittedly, the pedestrian load model presented in this paper may be less precise than laboratory-based models for describing single pedestrian behavior when walking on a moving platform and thus cannot fully represent the involved mechanism. However, given that laboratory-based models cannot be conveniently used in algebraic analysis, in addition to their unconfirmed application to crowd action on a bridge, 
the pedestrian load model adopted in this study appears at least justifiable for meeting the requirements of engineering design. It is worth emphasizing that the aim of this study is to establish an algebraic framework of nonlinear stochastic vibration for footbridge's lateral vibration. We believe that the application of this framework will not be limited to the current pedestrian load model. Nevertheless, there are also some other limitations of the proposed model. This paper considers only the first harmonic of pedestrian lateral load and ignores the higher harmonics. Meanwhile, for the sake of simplicity, this paper does not consider the intersubject variability among crowds and assumes that the synchronized pedestrians are identical. Moreover, although the proposed model, as a kind of macroscopic model, is supported by direct evidence on full-scale bridges and is suitable for engineering applications, its reliability still needs further study because the involved parameters are empirical and the amount of convincing measurement data remains insufficient. Therefore, additional measurement data and highly precise analyses are required to validate this model further.

\section{Conclusions}

The main conclusions can be summarized as follows:

(1) Unlike the numerical simulation method that requires a large amount of calculation, the proposed model is established in an algebraic theoretic framework of nonlinear stochastic vibration.

(2) The predicted results based on the proposed model show good agreement with the test observations from the M-Bridge and the P-Bridge.

(3) The frequency distribution significantly influences the stability of vibration. The worst condition takes place when the central lateral walking frequency is equal to the doubled first lateral frequency of bridge (i.e., when parametric resonance happens). In such case, the vibration stability reaches the lowest level while its sensitivity to the number of pedestrians and the random disturbance intensity reaches the highest level.

(4) The number of pedestrians and random disturbance intensity also greatly influence the stability. Increasing the number of pedestrians will decrease the stability and make the stability more sensitive to the distribution of frequency. The influence of random disturbance intensity on the stability depends on the frequency difference between the pedestrian and bridge. When the central lateral walking frequency approaches the doubled first lateral frequency of the bridge, an increase in the random disturbance intensity will increase the stability and make the stability less sensitive to the distribution of frequency. However, when the central lateral walking frequency deviates from the doubled first lateral frequency of the bridge, the random disturbance intensity has a relatively small influence on the stability and its increase leads to a slight decrease of the stability.

\section{Appendix}

\section{Equivalent Conversion of PSD}

The Gaussian-shaped $S_{F}(f)$ of the pedestrian lateral excitation process as obtained from the test results of Pizzimenti and Ricciardelli [22] takes the following form:

$$
\frac{S_{F}(f)}{\sigma_{F}^{2}}=\frac{1}{f} \frac{a_{s}}{\sqrt{2 \pi} b_{s}} \exp \left\{\left[-2\left(\frac{f / f_{p}-1}{b_{s}}\right)\right]^{2}\right\},
$$

where $\sigma_{F}^{2}=W^{2} d^{2}$ ( $W$ is the single pedestrian weight and $d$ is the dynamic loading factor) denotes the doubled area of PSD around the first load harmonic, $f_{p}$ denotes the pedestrian walking frequency, and $a_{s}=0.9$ and $b_{s}=$ 0.043 are the fitting parameters. To facilitate the following nonlinear stochastic equations, the Gaussian-shaped PSD is converted into a rational form that is expressed by the harmonic function. Equation (A.1) is changed into a function of angular frequency as follows:

$$
S_{F}(\omega)=\frac{\sigma_{F}^{2} \sqrt{2 \pi}}{\omega} \frac{a_{s}}{b_{s}} \exp \left\{\left[-2\left(\frac{\omega / \omega_{p}-1}{b_{s}}\right)\right]^{2}\right\},
$$

supporting the fact that the equivalent rational PSD has the following form:

$$
S_{F}^{*}(\omega)=\frac{\sigma_{F}^{* 2}}{2 \pi} \frac{\eta}{\left(\omega_{p}-\omega\right)^{2}+\eta^{2}} .
$$

Assuming that the peak values and curvatures of (A.2) and (A.3) are equal when $\omega=\omega_{p}$, we obtain the following:

$$
\begin{aligned}
& S_{F}\left(\omega_{p}\right)=S_{F}^{*}\left(\omega_{p}\right), \\
& S_{F}^{\prime \prime}\left(\omega_{p}\right)=S_{F}^{\prime \prime *}\left(\omega_{p}\right) .
\end{aligned}
$$

According to (A.4), we obtain

$$
\begin{aligned}
\eta & =\frac{\omega_{p} b_{s}}{\sqrt{2-b_{s}^{2}}}, \\
\sigma_{F}^{* 2} & =h^{2} \sigma_{F}^{2},
\end{aligned}
$$

where $h=\sqrt{2 \pi \sqrt{2 \pi} a_{s} / \sqrt{2-b_{s}^{2}}}$. Based on (A.5), (A.3) is further changed into a two-sided spectrum as follows:

$$
\begin{aligned}
S_{F}^{* *}(\omega) & =\frac{\sigma_{F}^{* 2}}{2}\left[S_{F}^{*}(\omega)+S_{F}^{*}(-\omega)\right] \\
& =h^{2} \sigma_{F}^{2} \frac{\eta}{2 \pi} \frac{\omega_{p}^{2}+\omega^{2}+\eta^{2}}{\left(\omega_{p}^{2}-\omega^{2}+\eta^{2}\right)^{2}+4 \eta^{2} \omega^{2}} .
\end{aligned}
$$

On the other hand, a narrowband process with a rational spectrum can be generated by a harmonic function with a random frequency and phase as shown in (5). The PSD of 
$\xi(t)$ can be obtained as follows via the Fourier transform of the covariance function:

$$
S_{F}(\omega)=\sigma_{\xi}^{2} \frac{\delta^{2}}{4 \pi} \frac{\omega_{p}^{2}+\omega^{2}+\delta^{4} / 4}{\left(\omega_{p}^{2}-\omega^{2}+\delta^{4} / 4\right)^{2}+\delta^{4} \omega^{2}} .
$$

By comparing (A.6) and (A.7), both of these equations become equal when $\delta=\sqrt{2 \eta}$ and $\sigma_{\xi}=h \sigma_{F}$. Finally, the equivalent harmonic function for representing the pedestrian lateral excitation process is obtained as shown in (6).

\section{Conflicts of Interest}

The authors declare that there are no conflicts of interest regarding the publication of this manuscript.

\section{Acknowledgments}

This research was supported by the National Natural Science Foundation of China (nos. 51478193 and 51608207), the China Postdoctoral Science Foundation (no. 2016M592490), the Fundamental Research Funds for the Central Universities (no. 2015ZM114), and the Open Fund of State Key Laboratory of Bridge Engineering Structural Dynamics (no. 201507).

\section{References}

[1] Y. Fujino, B. M. Pacheco, S.-I. Nakamura, and P. Warnitchai, "Synchronization of human walking observed during lateral vibration of a congested pedestrian bridge," Earthquake Engineering and Structural Dynamics, vol. 22, no. 9, pp. 741-758, 1993.

[2] P. Dallard, A. J. Fitzpatrick, A. Flint et al., "The London millennium footbridge," Structural Engineer, vol. 79, no. 22, pp. $17-33,2001$.

[3] S.-I. Nakamura, "Model for lateral excitation of footbridges by synchronous walking," Journal of Structural Engineering, vol. 130, no. 1, pp. 32-37, 2004.

[4] E. T. Ingólfsson and C. T. Georgakis, "A stochastic load model for pedestrian-induced lateral forces on footbridges," Engineering Structures, vol. 33, no. 12, pp. 3454-3470, 2011.

[5] E. T. Ingólfsson, C. T. Georgakis, F. Ricciardelli, and J. Jönsson, "Experimental identification of pedestrian-induced lateral forces on footbridges," Journal of Sound and Vibration, vol. 330, no. 6, pp. 1265-1284, 2011.

[6] J. H. Macdonald, "Lateral excitation of bridges by balancing pedestrians," Proceedings of The Royal Society of London. Series A. Mathematical, Physical and Engineering Sciences, vol. 465, no. 2104, pp. 1055-1073, 2009.

[7] M. Bocian, J. H. G. MacDonald, and J. F. Burn, "Biomechanically inspired modelling of pedestrian-induced forces on laterally oscillating structures," Journal of Sound and Vibration, vol. 331, no. 16, pp. 3914-3929, 2012.

[8] T. M. Roberts, "Lateral pedestrian excitation of footbridges," Journal of Bridge Engineering, vol. 10, no. 1, pp. 107-112, 2005.

[9] D. E. Newland, "Pedestrian excitation of bridges," Proceedings of the Institution of Mechanical Engineers, Part C: Journal of Mechanical Engineering Science, vol. 218, no. 5, pp. 477-492, 2004.
[10] G. Piccardo and F. Tubino, "Parametric resonance of flexible footbridges under crowd-induced lateral excitation," Journal of Sound and Vibration, vol. 311, no. 1-2, pp. 353-371, 2008.

[11] A. N. Blekherman, "Swaying of pedestrian bridges," Journal of Bridge Engineering, vol. 10, no. 2, pp. 142-150, 2005.

[12] A. N. Blekherman, "Autoparametric resonance in a pedestrian steel arch bridge: solferino bridge, Paris," Journal of Bridge Engineering, vol. 12, no. 6, pp. 669-676, 2007.

[13] S. H. Strogatz, D. M. Abrams, A. McRobie, B. Eckhardt, and E. Ott, "Crowd synchrony on the Millennium Bridge," Nature, vol. 438, no. 7064, pp. 43-44, 2005.

[14] J. M. W. Brownjohn, A. Pavic, and P. A. Omenzetter, "A spectral density approach for modelling continuous vertical forces on pedestrian structures due to walking," Canadian Journal of Civil Engineering, vol. 31, no. 1, pp. 65-77, 2004.

[15] S. Živanović, A. Pavić, and P. Reynolds, "Probability-based prediction of multi-mode vibration response to walking excitation," Engineering Structures, vol. 29, no. 6, pp. 942-954, 2007.

[16] V. Racic and J. M. W. Brownjohn, "Mathematical modelling of random narrow band lateral excitation of footbridges due to pedestrians walking," Computers and Structures, vol. 90-91, no. 1, pp. 116-130, 2012.

[17] V. Racic and J. M. W. Brownjohn, "Stochastic model of nearperiodic vertical loads due to humans walking," Advanced Engineering Informatics, vol. 25, no. 2, pp. 259-275, 2011.

[18] Z. Bin and X. Weiping, "A nonlinear analysis for the lateral girder response of footbridges induced by pedestrians," in Proceedings of the 2011 International Conference on Electric Technology and Civil Engineering, ICETCE 2011, pp. 4743-4746, 2011.

[19] X. B. Yuan, Research on Pedestrian-Induced Vibration of Footbridge [Ph.D. thesis], Tongji University, Shanghai, China, 2006.

[20] A. Ebrahimpour, A. Hamam, R. L. Sack, and W. N. Patten, "Measuring and modeling dynamic loads imposed by moving crowds," Journal of Structural Engineering, vol. 122, no. 12, pp. 1468-1474, 1996.

[21] M. Shinozuka and G. Deodatis, "Simulation of stochastic processes by spectral representation," Applied Mechanics Reviews, vol. 44, no. 4, pp. 191-204, 1991.

[22] A. Pizzimenti and F. Ricciardelli, "Experimental evaluation of the dynamic lateral loading of footbridges by walking pedestrians," in Proceedings of the 6th international conference on structural dynamics, Paris, France, 2005.

[23] P. Dallard, T. Fitzpatrick, A. Flint et al., "London millennium bridge: pedestrian-induced lateral vibration," Journal of Bridge Engineering, vol. 6, no. 6, pp. 412-417, 2001.

[24] R. Hai-wu, X. Wei, W. Xiang-dong, M. Guang, and F. Tong, "Principal response of van der pol-duffing oscillator under combined deterministic and random parametric excitation," Applied Mathematics and Mechanics, vol. 23, no. 3, pp. 299-310, 2002.

[25] E. T. Ingólfsson, C. T. Georgakis, and J. Jönsson, "Pedestrianinduced lateral vibrations of footbridges: a literature review," Engineering Structures, vol. 45, pp. 21-52, 2012.

[26] F. Lamarre, Passerelle Simone de Beauvoir - Paris, 2007, Feichtinger Architectes.

[27] A. L. Hof, R. M. van Bockel, T. Schoppen, and K. Postema, "Control of lateral balance in walking. Experimental findings in normal subjects and above-knee amputees," Gait and Posture, vol. 25, no. 2, pp. 250-258, 2007. 
[28] S. P. Carroll, J. S. Owen, and M. F. M. Hussein, "Reproduction of lateral ground reaction forces from visual marker data and analysis of balance response while walking on a laterally oscillating deck," Engineering Structures, vol. 49, pp. 1034-1047, 2013.

[29] S. P. Carroll, J. S. Owen, and M. F. M. Hussein, "Experimental identification of the lateral human-structure interaction mechanism and assessment of the inverted-pendulum biomechanical model," Journal of Sound and Vibration, vol. 333, no. 22, pp. 5865-5884, 2014.

[30] M. Bocian, J. H. G. Macdonald, J. F. Burn, and D. Redmill, "Experimental identification of the behaviour of and lateral forces from freely-walking pedestrians on laterally oscillating structures in a virtual reality environment," Engineering Structures, vol. 105, pp. 62-76, 2015. 


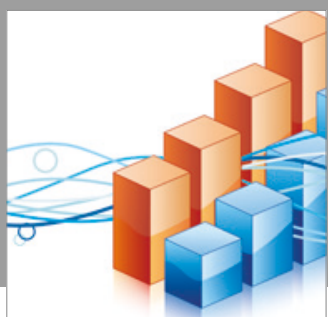

Advances in

Operations Research

vatersals

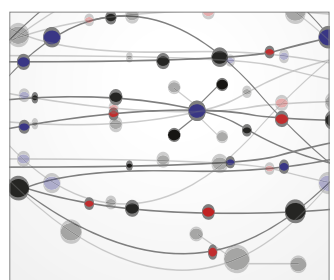

\section{The Scientific} World Journal
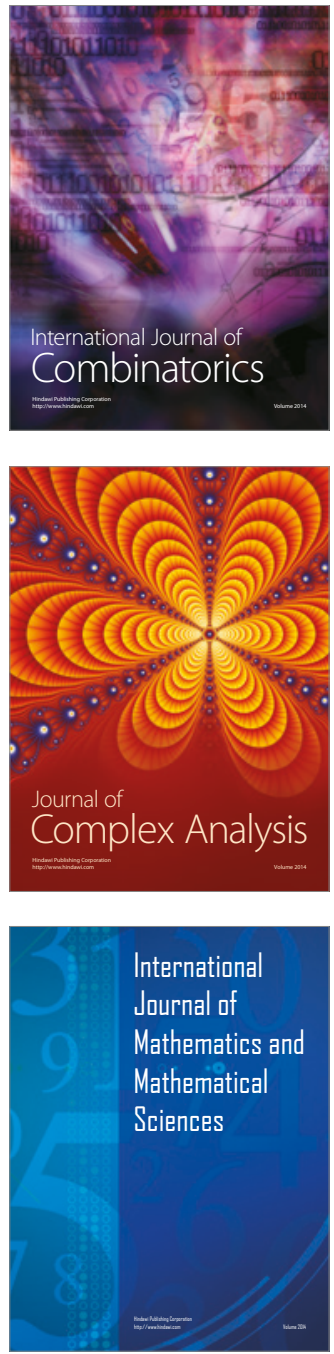
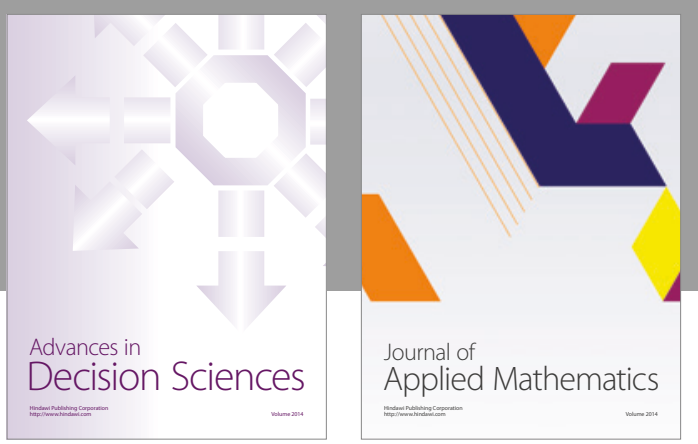

Algebra

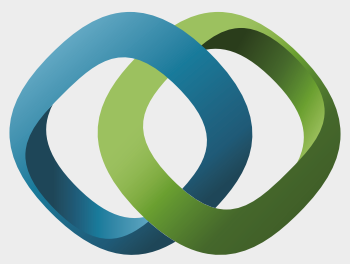

\section{Hindawi}

Submit your manuscripts at

https://www.hindawi.com
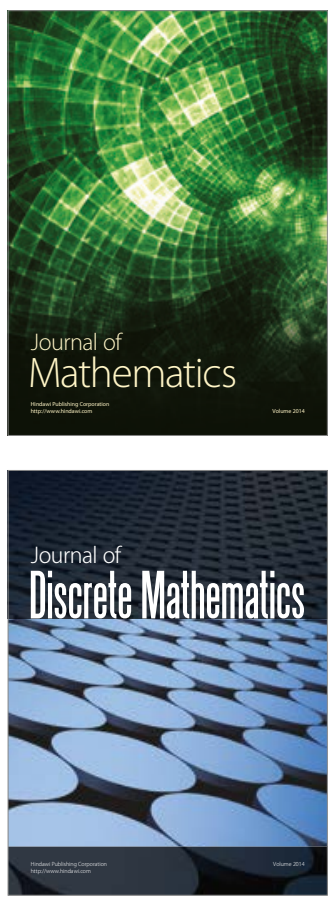

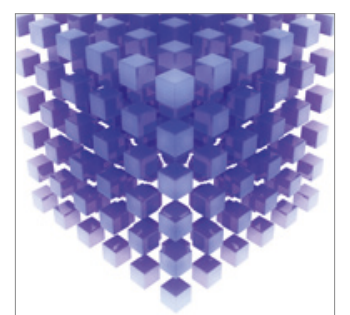

Mathematical Problems in Engineering
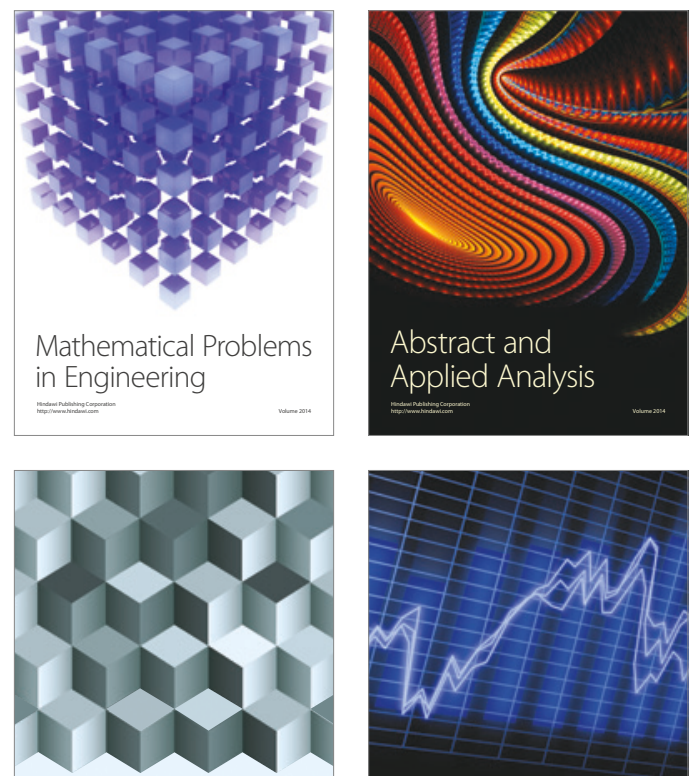

Journal of

Function Spaces

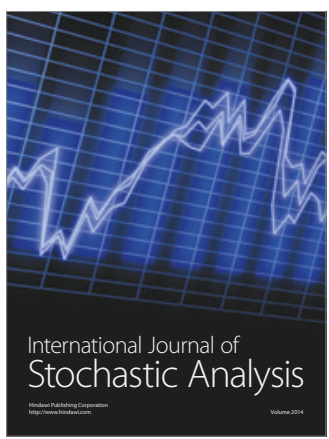

Probability and Statistics
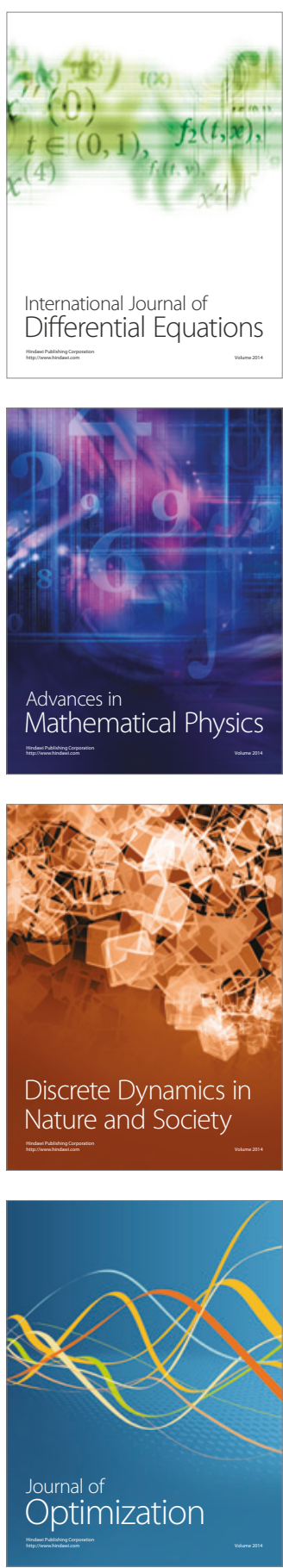